\title{
Restricted Real Perturbation Values with Applications to the Structured Real Controllability Radius of LTI Systems
}

\author{
Simon Lam and Edward J. Davison
}

\begin{abstract}
In this paper, the concept of restricted real perturbation values of a complex matrix triplet is introduced, and a formula for computing lower bounds of these values is presented. Restricted real perturbation values are a generalization of the real perturbation values introduced in [1], which is a key concept in evaluating various robustness radii found in the control literature, such as the real controllability/observability radius, the real decentralized fixed-mode radius, the real minimum-phase radius, etc. The generalization to restricted real perturbation values is needed for an extension of these radii to account for more general system perturbation structures. As an example, we will use the results of this paper to compute the true value of the structured real controllability radius of the multi-link inverted pendulum system. Also, we will numerically investigate cases of when the provided lower bounds are achievable.
\end{abstract}

\section{INTRODUCTION}

The real perturbation values of a complex matrix introduced in [1] is a key concept in computing the real controllability/observability radius ([2]), the real decentralized fixedmode radius ([3]), the real transmission zero at $s$ radius ([4]), and the minimum-phase radius ([4]) that are found in the control literature. This class of robustness radii measures the robustness of the various linear time-invariant (LTI) system properties in the presence of real parametric perturbations. In this paper, the concept of real perturbation values is extended to the restricted real perturbation values, which allows for more general perturbation structures to be considered when computing these various robustness radii.

To motivate the study of these robustness radii, consider the following LTI multivariable system

$$
\begin{aligned}
\dot{x} & =A x+B u \\
y & =C x+D u
\end{aligned}
$$

where $x \in \mathbb{R}^{n}, u \in \mathbb{R}^{m}$, and $y \in \mathbb{R}^{r}$ are respectively the states, inputs, and outputs of the system. Suppose the system is controllable and observable, then it is well known that there exists a LTI controller that can assign the eigenvalues of the closed-loop system to any desirable spectrum. However, if the system is subjected to real parametric perturbations (i.e. $A \rightarrow A+\Delta_{A}$ and $B \rightarrow B+\Delta_{B}$, where $\Delta_{A}$ and $\Delta_{B}$ are real matrices), which may result from numerical errors, modeling errors, etc., then the perturbed system may be uncontrollable. Hence, a continuous real controllability

This work has been supported by NSERC under grant No. A4396.

S. Lam and E. J. Davison are with the Systems Control Group, Department of Electrical and Computer Engineering, University of Toronto, Toronto, ON, Canada M5S 3G4 $\{$ simon, ted $\}$ control.utoronto.ca radius that can measure how "close" a controllable system is to an uncontrollable one is more informative than the traditional 'yes/no' controllability metric, which simply determines whether a system is controllable or not. The same motivation also applies to the other radii with respect to the various other LTI system properties.

The formulas for computing these radii are all based on solving the following perturbation problem: given a complex matrix $M$ and an integer $i>0$, find the size of the "smallest" perturbation, $\Delta$, such that

$$
\operatorname{rank}(M-\Delta)<i \quad \text { where } M \text { is complex }
$$

If $\Delta$ can be any complex matrix and the "size" is measured by the spectral norm, $\|\cdot\|_{2}$, then the solution of this problem is related to the well-known singular values of $M$. If $\Delta$ is constrained to be a real matrix, then the solution is related to [5], and to the real perturbation values of $M$ introduced in [1].

In a recent paper [6], the concept of real perturbation values is extended to the generalized real perturbation values, which are related to the generalized singular values in [7], [8], where both solve the perturbation problem (2) with an extended perturbation structure:

$$
\operatorname{rank}(M-\Delta N)<i \quad \text { where } M, N \text { are complex }
$$

In this paper, we will consider the problem (2) with an even more general perturbation structure, namely,

$$
\operatorname{rank}(M-L \Delta N)<i \quad \text { where } M, L, N \text { are complex }
$$

In view of the restricted singular values of a matrix triplet (see [9], [10]), which solves (4) for complex $\Delta$, we will consider (4) for the case when $\Delta$ is real with the introduction of the so-called restricted real perturbation values (named as such for obvious reasons).

This paper is organized as follows. In Section II, the concepts of real perturbation values and restricted singular values of a complex matrix are reviewed. Then in Section III, we define the restricted real perturbation values of a matrix triplet, and present our main result, which is a formula for computing lower bounds of these values. Finally in Section IV, we will revisit the pendulum problem in [11], and apply the results of this paper to compute the true value of the structured real controllability radius of the multi-link inverted pendulum. Also, we will numerically show that the lower bounds provided in this paper are actually achievable for the cases when $L$ (or $N$ ) has full column (or row) rank and/or is real. 


\section{PRELIMINARIES AND REVIEW}

\section{A. Notation}

In this paper, the field of real and complex numbers are denoted by $\mathbb{R}$ and $\mathbb{C}$ respectively. The $i$-th singular value of a matrix $M \in \mathbb{C}^{n \times m}$ is denoted by $\sigma_{i}(M)$, where $\sigma_{1}(M) \geq$ $\cdots \geq \sigma_{\min (n, m)}(M) .\|M\|_{2}$ denotes the spectral norm of $M$ and is equal to $\sigma_{1}(M)$. Also, $\bar{M}$ and $M^{*}$ denote respectively the complex conjugate, and the complex conjugate transpose of $M$. The real and imaginary components of $M$ are given by $\operatorname{Re} M$ and $\operatorname{Im} M$ respectively. Lastly, $M^{\mathbb{R}}$ denotes the representation of a complex matrix $M \in \mathbb{C}^{n \times m}$ in real form (e.g. see [12]); namely,

$$
M^{\mathbb{R}}=T_{n}\left[\begin{array}{cc}
M & 0 \\
0 & \bar{M}
\end{array}\right] T_{m}=\left[\begin{array}{cc}
\operatorname{Re} M & -\operatorname{Im} M \\
\operatorname{Im} M & \operatorname{Re} M
\end{array}\right]
$$

where we denote $T_{q}:=\frac{1}{\sqrt{2}}\left[\begin{array}{cc}I_{q} & i I_{q} \\ -i I_{q} & -I_{q}\end{array}\right]$, for $q \in \mathbb{N}$.

\section{B. Review of real perturbation values}

The real perturbation values introduced in [1] are defined as follows.

Definition 2.1 (Real perturbation values [1]): Given $M \in \mathbb{C}^{n \times m}$, the $i$-th real perturbation values ${ }^{1}$ of $M$, for $i=1, \ldots, \min (n, m)$, are defined as:

$$
\tau_{i}(M):=\inf _{\Delta \in \mathbb{R}^{n \times m}}\left\{\|\Delta\|_{2} \mid \operatorname{rank}(M-\Delta)<i\right\}
$$

Remark 2.1: Given a matrix $M \in \mathbb{C}^{n \times m}$, the singular values of $M$ satisfy (e.g. see [13]):

$$
\sigma_{i}(M)=\inf _{\Delta \in \mathbb{C}^{n \times m}}\left\{\|\Delta\|_{2} \mid \operatorname{rank}(M-\Delta)<i\right\}
$$

for $i=1, \ldots, \min (n, m)$. As mentioned earlier, the real perturbation values are closely related to the singular values. Here we see this more closely by comparing (6) with (7).

The real perturbation values can be computed by the following formula [1].

Theorem 2.1 ([1]): Given $M \in \mathbb{C}^{n \times m}$ and $i=$ $1, \ldots, \min (n, m)$

$$
\begin{aligned}
\tau_{i}(M) & =\sup _{\gamma \in(0,1]} \sigma_{2 i-1}\left(\left[\begin{array}{cc}
\operatorname{Re} M & -\gamma \operatorname{Im} M \\
\gamma^{-1} \operatorname{Im} M & \operatorname{Re} M
\end{array}\right]\right) \\
& =\sup _{\gamma \in(0,1]} \sigma_{2 i-1}\left(\left[\begin{array}{cc}
\gamma I & 0 \\
0 & I
\end{array}\right] M^{\mathbb{R}}\left[\begin{array}{cc}
\gamma^{-1} I & 0 \\
0 & I
\end{array}\right]\right)
\end{aligned}
$$

For ease of presentation, we will use the following notation in the remainder of the paper. Given $M^{\mathbb{R}}$, then:

$$
M_{\gamma}^{\mathbb{R}}:=\left[\begin{array}{cc}
\gamma I & 0 \\
0 & I
\end{array}\right] M^{\mathbb{R}}\left[\begin{array}{cc}
\gamma^{-1} I & 0 \\
0 & I
\end{array}\right]
$$

\footnotetext{
${ }^{1}$ The definition of the real perturbation values used in this paper corresponds to the real perturbation values of the second kind defined in [1].
}

\section{Review of restricted singular values}

The restricted singular values of a matrix triplet was introduced in [9].

Definition 2.2 (Restricted singular values [9]): Given $M \in \mathbb{C}^{n \times m}, L \in \mathbb{C}^{n \times l}$, and $N \in \mathbb{C}^{p \times m}$, the restricted singular values of the triplet $(M, L, N)$ are defined as:

$$
\sigma_{i}(M, L, N):=\inf _{\Delta \in \mathbb{C}^{l \times p}}\left\{\|\Delta\|_{2} \mid \operatorname{rank}(M-L \Delta N)<i\right\}
$$

for $i=1, \ldots, \min (n, m)$.

Remark 2.2: In this paper, $\sigma$ will be used to denote the singular values of a matrix, where it will be clear from the number of arguments which specific type it is referring to; i.e. $\sigma_{i}(M), \sigma_{i}(M, N)$, and $\sigma_{i}(M, L, N)$ refer to the ordinary, generalized, and restricted singular values, previously discussed in (2), (3), and (4) respectively.

The restricted singular values of a given matrix triplet can be obtained by the restricted singular value decomposition (see [9], [10]).

Theorem 2.2 (Restricted singular value decomposition): Given $M \in \mathbb{C}^{n \times m}, L \in \mathbb{C}^{n \times l}$, and $N \in \mathbb{C}^{p \times m}$, there exist nonsingular matrices $X \in \mathbb{C}^{n \times n}$ and $Y \in \mathbb{C}^{m \times m}$, and unitary matrices $U \in \mathbb{C}^{l \times l}$ and $V \in \mathbb{C}^{p \times p}$ such that

$$
\begin{aligned}
& \begin{array}{llll}
k_{1} & k_{2} & k_{3} & \mu
\end{array} \\
& \left.X^{-1} M Y^{-1}=k_{1}=\begin{array}{ccccc}
I & 0 & 0 & 0 & 0 \\
0 & I & 0 & 0 & 0 \\
k_{3} \\
\nu & 0 & I & 0 & 0 \\
0 & 0 & 0 & S_{M} & 0 \\
0 & 0 & 0 & 0 & 0
\end{array}\right]
\end{aligned}
$$

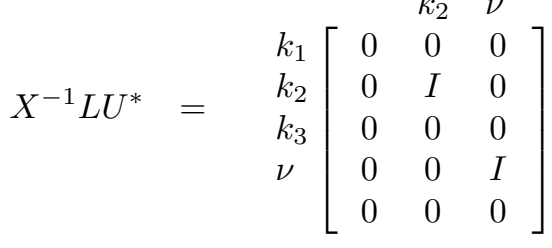

$$
\begin{aligned}
& V^{*} N Y^{-1}=k_{3}\left[\begin{array}{ccccc}
k_{1} & k_{2} & k_{3} & \mu & \\
0 & 0 & 0 & 0 & 0 \\
0 & 0 & I & 0 & 0 \\
0 & 0 & 0 & I & 0
\end{array}\right]
\end{aligned}
$$

where $S_{M}=\left[\begin{array}{ll}\Sigma & 0 \\ 0 & 0\end{array}\right]$ with $\Sigma$ being a real positive diagonal matrix.

From Theorem 2.2, it can be seen that the restricted singular values of a matrix triplet $(M, L, N)$ can be infinite, finite, or undefined (i.e. trivial). In particular, there are $k_{1}+k_{2}+k_{3}$ infinite restricted singular values, $\min (\nu, \mu)$ finite restricted singular values corresponding to the diagonal elements of $S_{M}$, and $\min \left(n-k_{1}-k_{2}-k_{3}-\nu, m-k_{1}-k_{2}-k_{3}-\mu\right) \quad$ trivial restricted singular values.

\section{MAIN RESULT}

\section{A. Restricted real perturbation values}

The following generalization is made.

Definition 3.1 (Restricted real perturbation values):

Given $M \in \mathbb{C}^{n \times m}, L \in \mathbb{C}^{n \times l}$, and $N \in \mathbb{C}^{p \times m}$, the $i$-th 
restricted real perturbation value of the matrix triplet $(M, L, N)$ is defined as:

$$
\tau_{i}(M, L, N):=\inf _{\Delta \in \mathbb{R}^{l \times p}}\left\{\|\Delta\|_{2} \mid \operatorname{rank}(M-L \Delta N)<i\right\}
$$

where $i=1, \ldots, \min (n, m)$. If there exists no real matrix $\Delta$ such that $\operatorname{rank}(M-L \Delta N)<i$, then $\tau_{i}(M, L, N)=\infty$.

In terms of notation, $\tau$ will be used to denote the real perturbation values of a matrix, and again it will be clear from the number of arguments which type it is referring to (e.g. see Remark 2.2); namely, $\tau_{i}(M), \tau_{i}(M, N)$, and $\tau_{i}(M, L, N)$ refer to the ordinary, generalized, and restricted real perturbation values respectively.

Remark 3.1: If $L$ and $N$ are nonsingular, then the restricted real perturbation value problem can be reduced to the ordinary real perturbation value problem (see Definition 2.1) of the single matrix $L^{-1} M N^{-1}$. This is because

$$
\operatorname{rank}(M-L \Delta N)=\operatorname{rank}\left(L^{-1} M N^{-1}-\Delta\right)
$$

Similarly, if only $L$ is nonsingular, then the problem can be reduced to the generalized real perturbation value problem of the matrix pair $\left(L^{-1} M, N\right)$ (see [6]). In this paper, $L$ and $N$ will be general matrices with no assumptions on invertibility.

\section{B. Lower bounds of the restricted real perturbation values}

The following theorem presents a formula for computing lower bounds of the restricted real perturbation values of a matrix triplet.

Theorem 3.1: Given $M \in \mathbb{C}^{n \times m}, L \in \mathbb{C}^{n \times l}, N \in \mathbb{C}^{p \times m}$, and $i=1, \ldots, \min (n, m)$, then

$$
\tau_{i}(M, L, N) \geq \sup _{\gamma \in(0,1]} \sigma_{2 i-1}\left(M_{\gamma}^{\mathbb{R}}, L_{\gamma}^{\mathbb{R}}, N_{\gamma}^{\mathbb{R}}\right)
$$

where $M_{\gamma}^{\mathbb{R}}, L_{\gamma}^{\mathbb{R}}$ and $N_{\gamma}^{\mathbb{R}}$ are defined by (9).

Proof: We will prove Theorem 3.1 by showing that

$$
\tau_{i}(M, L, N) \leq \tau \Rightarrow \sup _{\gamma \in(0,1]} \sigma_{2 i-1}\left(M_{\gamma}^{\mathbb{R}}, L_{\gamma}^{\mathbb{R}}, N_{\gamma}^{\mathbb{R}}\right) \leq \tau
$$

is true for any real $\tau \geq 0$ and $i=1, \ldots, \min (n, m)$; i.e. if $\tau_{i}(M, L, N) \leq \tau$, then there exists $\Delta \in \mathbb{R}^{l \times p}$ such that $\|\Delta\|_{2} \leq \tau$ and $\operatorname{rank}(M-L \Delta N)<i$, or equivalently,

$$
\operatorname{rank}\left(M^{\mathbb{R}}-L^{\mathbb{R}}\left[\begin{array}{cc}
\Delta & 0 \\
0 & \Delta
\end{array}\right] N^{\mathbb{R}}\right)<2 i-1
$$

where the inequality is true because the rank of a matrix belongs to the set of nonnegative integers. Pre- and postmultiplying the left-hand side of (13) by $\left[\begin{array}{cc}\gamma I & 0 \\ 0 & I\end{array}\right]$ and $\left[\begin{array}{cc}\gamma^{-1} I & 0 \\ 0 & I\end{array}\right]$ respectively, for $\gamma \in(0,1]$, we obtain:

$$
\operatorname{rank}\left(M_{\gamma}^{\mathbb{R}}-L_{\gamma}^{\mathbb{R}}\left[\begin{array}{cc}
\Delta & 0 \\
0 & \Delta
\end{array}\right] N_{\gamma}^{\mathbb{R}}\right)<2 i-1
$$

Hence $\left[\begin{array}{cc}\Delta & 0 \\ 0 & \Delta\end{array}\right]$ is a perturbation to $\left(M_{\gamma}^{\mathbb{R}}, L_{\gamma}^{\mathbb{R}}, N_{\gamma}^{\mathbb{R}}\right)$. So recalling the definition of restricted singular values (i.e. Definition 2.2), we see that

$$
\sigma_{2 i-1}\left(M_{\gamma}^{\mathbb{R}}, L_{\gamma}^{\mathbb{R}}, N_{\gamma}^{\mathbb{R}}\right) \leq\left\|\left[\begin{array}{cc}
\Delta & 0 \\
0 & \Delta
\end{array}\right]\right\|_{2}=\|\Delta\|_{2} \leq \tau
$$

for $\gamma \in(0,1]$. Hence (12) follows immediately.

Remark 3.2: Since the set of real matrices is a subset of the set of complex matrices, one can see from Definition 2.2 and 3.1 that the restricted singular values of $(M, L, N)$ can also be used as lower bounds for the restricted real perturbation values of $(M, L, N)$; i.e. for $i=1, \ldots, \min (n, m)$,

$$
\tau_{i}(M, L, N) \geq \sigma_{i}(M, L, N)
$$

The following theorem shows that the lower bounds provided by Theorem 3.1 are in fact tighter than the lower bounds given in (14), and we will see in the second example of Section IV that the difference can indeed be quite large.

Theorem 3.2: Given $M \in \mathbb{C}^{n \times m}, L \in \mathbb{C}^{n \times l}, N \in \mathbb{C}^{p \times m}$, and $i=1, \ldots, \min (n, m)$,

$$
\sigma_{i}(M, L, N) \leq \sup _{\gamma \in(0,1]} \sigma_{2 i-1}\left(M_{\gamma}^{\mathbb{R}}, L_{\gamma}^{\mathbb{R}}, N_{\gamma}^{\mathbb{R}}\right)
$$

Proof: Let the following be true for any real $\tau \geq 0$ and $i=1, \ldots, \min (n, m)$ :

$$
\sup _{\gamma \in(0,1]} \sigma_{2 i-1}\left(M_{\gamma}^{\mathbb{R}}, L_{\gamma}^{\mathbb{R}}, N_{\gamma}^{\mathbb{R}}\right) \leq \tau
$$

Then, $\sigma_{2 i-1}\left(M^{\mathbb{R}}, L^{\mathbb{R}}, N^{\mathbb{R}}\right) \leq \tau$, or equivalently, there exists $\Delta \in \mathbb{C}^{2 l \times 2 p}$ such that $\|\Delta\|_{2} \leq \tau$ and $\operatorname{rank}\left(M^{\mathbb{R}}-L^{\mathbb{R}} \Delta N^{\mathbb{R}}\right)<2 i-1$. By (5), this is equivalent to

$$
\left.\operatorname{rank}\left(\left[\begin{array}{cc}
M & 0 \\
0 & \bar{M}
\end{array}\right]-\left[\begin{array}{cc}
L & 0 \\
0 & \bar{L}
\end{array}\right] T_{l} \Delta T_{p}\left[\begin{array}{cc}
N & 0 \\
0 & \bar{N}
\end{array}\right]\right)\right)_{2 i-1}
$$

Since $T_{l}$ and $T_{p}$ are unitary, then $\left\|T_{l} \Delta T_{p}\right\|_{2}=\|\Delta\|_{2} \leq \tau$. Therefore,

$$
\sigma_{2 i-1}\left(\left[\begin{array}{cc}
M & 0 \\
0 & \bar{M}
\end{array}\right],\left[\begin{array}{cc}
L & 0 \\
0 & \bar{L}
\end{array}\right],\left[\begin{array}{cc}
N & 0 \\
0 & \bar{N}
\end{array}\right]\right) \leq \tau
$$

which implies that $\sigma_{i}(M, L, N) \leq \tau$.

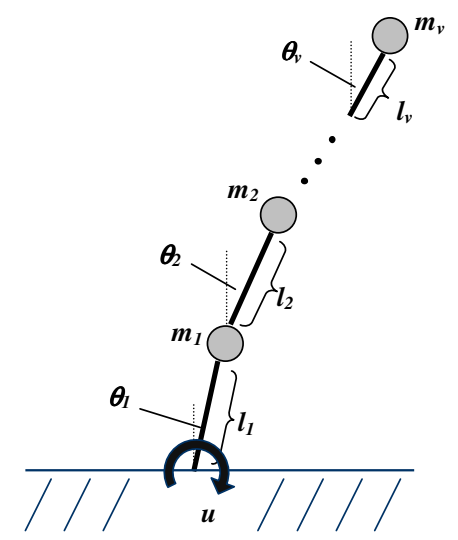

Fig. 1. Model of a multi-link inverted pendulum with $v$ links ([11]).

\section{APPLICATIONS}

In this section, we will use the restricted real perturbation values to study two numerical examples. First, we will revisit the pendulum problem in [11] and using the results of this paper, compute a lower bound of the true structured real 
controllability radius of the multi-link inverted pendulum. In the second example, we will study cases of when the lower bounds provided by Theorem 3.1 are actually achievable.

\section{A. Structured real controllability radius of the pendulum}

In [11], the real controllability radius was used to study the difficulty of balancing the multi-link inverted pendulum system with $v$ links (see Figure 1), for $v=1,2, \ldots$, whose linear state space model is given in [11] as:

$$
\begin{aligned}
& A=\left[\begin{array}{cc}
0 & I \\
\left(M_{v} L_{v}\right)^{-1} M_{a} & 0
\end{array}\right], B=\left[\begin{array}{c}
0 \\
\left(M_{v} L_{v}\right)^{-1} M_{b}
\end{array}\right] \\
& C=\left[\begin{array}{llll}
1 & 0 & \ldots & 0
\end{array}\right] \quad, D=0
\end{aligned}
$$

where $M_{a}=\operatorname{diag}\left(g \sum_{i=1}^{v} m_{i}, g \sum_{i=2}^{v} m_{i}, \cdots, m_{v} g\right), M_{b}=$ $\left[\begin{array}{c}\frac{1}{l_{1}} \\ 0 \\ \vdots \\ \vdots\end{array}\right], M_{v}=\left[\begin{array}{cccc}m_{1} & m_{2} & \cdots & m_{v} \\ 0 & m_{2} & \cdots & m_{v} \\ \vdots & \vdots & \ddots & \vdots \\ 0 & 0 & \cdots & m_{v}\end{array}\right]$, and $L_{v}=\left[\begin{array}{cccc}l_{1} & 0 & \cdots & 0 \\ l_{1} & l_{2} & \cdots & 0 \\ \vdots & \vdots & \ddots & 0 \\ l_{1} & l_{2} & \cdots & l_{v}\end{array}\right]$. In particular, it was claimed in [11] that the difficulty is related to the pendulum's controllability robustness, and it was numerically shown that as the number of links increases, the radius becomes smaller, indicating that the system becomes almost uncontrollable.

The system perturbation structure considered in [11] was of the following form:

$$
\begin{aligned}
A & \rightarrow\left(I+\left[\begin{array}{ll}
0 & 0 \\
0 & I
\end{array}\right] \Delta_{A}\right) A \\
B & \rightarrow\left(I+\left[\begin{array}{ll}
0 & 0 \\
0 & I
\end{array}\right] \Delta_{B}\right) B
\end{aligned}
$$

which led to a structured controllability radius problem that was difficult to solve at the time. Subsequently, only an estimate of the actual radius was obtained in [11]. In this section, we will use the main results of this paper to study the true value of the inverted pendulum system's structured real controllability radius.

In [11], the structured real controllability radius of $A \in$ $\mathbb{R}^{n \times n}, B \in \mathbb{R}^{n \times m}, \mathcal{E} \in \mathbb{R}^{n \times l}, \mathcal{F} \in \mathbb{R}^{p \times n}$, and $\mathcal{G} \in \mathbb{R}^{q \times m}$ is defined to be:

$$
\begin{aligned}
r_{\mathbb{R}}^{c, s t r u c t} & (A, B, \mathcal{E}, \mathcal{F}, \mathcal{G}) \\
= & \inf \left\{\left\|\left[\Delta_{A}, \Delta_{B}\right]\right\|_{2} \mid \Delta_{A} \in \mathbb{R}^{l \times p}, \Delta_{B} \in \mathbb{R}^{l \times q},\right. \\
& \left.\left(A+\mathcal{E} \Delta_{A} \mathcal{F}, B+\mathcal{E} \Delta_{B} \mathcal{G}\right) \text { is uncontrollable }\right\}
\end{aligned}
$$

Hence the structured controllability radius of the pendulum system (16) with the perturbation structure (17) is given by (18), where

$$
\mathcal{E}=\left[\begin{array}{ll}
0 & 0 \\
0 & I
\end{array}\right], \quad \mathcal{F}=A, \quad \text { and } \quad \mathcal{G}=B
$$

In [11], the formula derived to compute $r_{\mathbb{R}}^{c, \text { struct }}$ is:

$$
\begin{aligned}
r_{\mathbb{R}}^{c, s t r u c t} & (A, B, \mathcal{E}, \mathcal{F}, \mathcal{G}) \\
= & \min _{s \in \mathbb{C}} \tau_{n}\left(\mathcal{E}^{-1}[A-s I, \quad B]\left[\begin{array}{cc}
\mathcal{F} & 0 \\
0 & \mathcal{G}
\end{array}\right]^{-1}\right)
\end{aligned}
$$

which is valid only for the case when $\mathcal{E}, \mathcal{F}$, and $\mathcal{G}$ are nonsingular matrices (this allowed the structured real controllability radius to be reduced to the unstructured case - see Remark 3.1). However, since $\mathcal{E}, \mathcal{F}$, and $\mathcal{G}$ in (19) are singular, only an estimate of the radius was obtained in [11] using (20). This was done by approximating $\mathcal{E}$ with a nonsingular matrix $\tilde{\mathcal{E}}=\left[\begin{array}{cc}\epsilon I & 0 \\ 0 & I\end{array}\right]$, where $\epsilon>0$ is chosen as small as possible (e.g. $\epsilon=10^{-6}$ ) such that $\tilde{\mathcal{E}}$ is not ill-conditioned, and also by approximating $\left[\begin{array}{cc}\mathcal{F} & 0 \\ 0 & \mathcal{G}\end{array}\right]$ with another nonsingular matrix via an ad-hoc normalization technique involving a random matrix.

In [6], the nonsingularity restriction of $\mathcal{F}$ and $\mathcal{G}$ in (20) was removed to obtain the following formula involving generalized real perturbation values:

$$
\begin{aligned}
& r_{\mathbb{R}}^{c, \text { struct }}(A, B, \mathcal{E}, \mathcal{F}, \mathcal{G}) \\
& \quad=\min _{s \in \mathbb{C}} \tau_{n}\left(\mathcal{E}^{-1}\left[\begin{array}{ll}
A-s I, & B
\end{array}\right],\left[\begin{array}{cc}
\mathcal{F} & 0 \\
0 & \mathcal{G}
\end{array}\right]\right)
\end{aligned}
$$

where $\mathcal{E}$ is still required to be nonsingular. Hence, the results obtained in [6] using (21) were also only an estimate of the radius (obtained by approximating $\mathcal{E}$ by $\tilde{\mathcal{E}}=\left[\begin{array}{cc}\epsilon I & 0 \\ 0 & I\end{array}\right]$ ).

Using the results of this paper, we can now remove the final nonsingularity restriction of $\mathcal{E}$, and present a formula to compute $r_{\mathbb{R}}^{c \text { struct }}$ in (18) for all general $\mathcal{E}, \mathcal{F}$, and $\mathcal{G}$.

Theorem 4.1: Given the LTI system (1), and general matrices $\mathcal{E}, \mathcal{F}$, and $\mathcal{G}$ of conformal dimensions, the structured real controllability radius (18) is given as follows:

$$
\begin{aligned}
& r_{\mathbb{R}}^{c, \text { struct }}(A, B, \mathcal{E}, \mathcal{F}, \mathcal{G}) \\
&=\min _{s \in \mathbb{C}} \tau_{n}\left(\left[\begin{array}{ll}
A-s I, & B
\end{array}\right], \mathcal{E},\left[\begin{array}{cc}
\mathcal{F} & 0 \\
0 & \mathcal{G}
\end{array}\right]\right)
\end{aligned}
$$

Proof: The proof is straightforward. Given $s \in \mathbb{C}$, the perturbed system $\left(A+\mathcal{E} \Delta_{A} \mathcal{F}, B+\mathcal{E} \Delta_{B} \mathcal{G}\right)$ is uncontrollable at $s$ if and only if

$$
\operatorname{rank}\left(\left[A+\mathcal{E} \Delta_{A} \mathcal{F}-s I, B+\mathcal{E} \Delta_{B} \mathcal{G}\right]\right)<n
$$

or equivalently,

$$
\operatorname{rank}\left([A-s I, B]+\mathcal{E}\left[\Delta_{A}, \Delta_{B}\right]\left[\begin{array}{cc}
\mathcal{F} & 0 \\
0 & \mathcal{G}
\end{array}\right]\right)<n
$$

Therefore the smallest perturbations, $\left[\Delta_{A}, \Delta_{B}\right]$, such that the perturbed system is uncontrollable at $s$ is given by $\tau_{n}\left([A-s I, B], \mathcal{E},\left[\begin{array}{cc}\mathcal{F} & 0 \\ 0 & \mathcal{G}\end{array}\right]\right)$. The structured real controllability radius, $r_{\mathbb{R}}^{c, s t r u c t}$, is then the minimization of this function over $s$ in the complex plane.

Using Theorem 3.1, we computed lower bounds of the true structured controllability radius given by (22) of the pendulum system with 1 to 7 links, where each link is of unit mass and unit length (i.e. $m_{i}=1$ and $l_{i}=1$, for $i=1, \ldots, v)$. The results are listed in Table I, along with the values obtained in [11] and [6]. Here we see that the values obtained in [6] are almost equal (up to the $11^{\text {th }}$ significant figure) to the lower bound obtained by Theorem 3.1. Since one may expect $\tilde{\mathcal{E}}=\left[\begin{array}{cc}\epsilon I & 0 \\ 0 & I\end{array}\right]$ to be a good approximation of $\mathcal{E}=\left[\begin{array}{ll}0 & 0 \\ 0 & I\end{array}\right]$ for small $\epsilon$, this suggests that for the particular perturbation structure (17), the lower bound of the controllability radius is achievable, and that the values obtained in [6] via (21) are very close to the true radius. 
TABLE I

THE STRUCTURED REAL CONTROLLABILITY RADIUS OF A MULTI-LINK INVERTED PENDULUM SYSTEM (REVISITED)

\begin{tabular}{l|ccc}
\hline \hline$v$ & $r_{\mathbb{R}}^{c, \text { struct }}$ & $r_{\mathbb{R}}^{c, \text { struct }}$ & $r_{\mathbb{R}}^{c, \text { struct }}$ \\
& via (20) in [11] & via (21) in [6] & via (22)* \\
\hline 1 & $1.000_{10^{+0}}$ & $1.000_{10^{+0}}$ & $1.000_{10^{+0}}$ \\
2 & $1.111_{10^{-1}}$ & $1.085_{10^{-1}}$ & $1.085_{10^{-1}}$ \\
3 & $4.688_{10^{-2}}$ & $4.655_{10^{-2}}$ & $4.655_{10^{-2}}$ \\
4 & $2.456_{10^{-2}}$ & $2.450_{10^{-2}}$ & $2.450_{10^{-2}}$ \\
5 & $1.467_{10^{-2}}$ & $1.466_{10^{-2}}$ & $1.466_{10^{-2}}$ \\
6 & $9.565_{10^{-3}}$ & $9.559_{10^{-3}}$ & $9.559_{10^{-3}}$ \\
7 & $6.635_{10^{-3}}$ & $6.633_{10^{-3}}$ & $6.633_{10^{-3}}$ \\
\hline \hline
\end{tabular}

* These values are in fact lower bounds of (22) obtained by using Theorem 3.1.

\section{B. Study of when the lower bound (11) is achievable}

In the previous example, we saw that we are able to obtain an estimate of the structured real controllability radius that is very close to the lower bound provided by Theorem 3.1, which suggests that the lower bound is achievable. In this example, we will investigate this further, namely, when does the restricted real perturbation values of a matrix triplet equal the lower bounds provided by Theorem 3.1 (i.e. (11))?

Similar to the approach used in the previous example, we will compute estimates of the restricted real perturbation values of $(M, L, N)$ by computing the generalized real perturbation values [6] of $\left(\tilde{L}^{-1} \tilde{M}, N\right)$; i.e. (see [6])

$$
\tau_{i}\left(\tilde{L}^{-1} \tilde{M}, N\right)=\sup _{\gamma \in(0,1]} \sigma_{2 i-1}\left(\left(\tilde{L}^{-1} \tilde{M}\right)^{\mathbb{R}}, N^{\mathbb{R}}\right)
$$

where $\tilde{L}$ is a nonsingular approximation of $L$, and $\tilde{M}=$ $\left[\begin{array}{cc}M & 0 \\ 0 & 0\end{array}\right]$ is equal to $M$ padded with zero columns/rows to conform with the dimensions of $\tilde{L}$ and $N$.

In order to be able to approximate a general matrix $L$ with a (square) nonsingular matrix, it should be noted that the matrices $(M, L, N)$ in Definition 3.1 can all be assumed, without loss of generality, to be square matrices with the same dimensions. This is because

$$
\begin{aligned}
& \operatorname{rank}(M-L \Delta N)= \\
& \operatorname{rank}\left(\left[\begin{array}{cc}
M & 0 \\
0 & 0
\end{array}\right]-\left[\begin{array}{cc}
L & 0 \\
0 & 0
\end{array}\right]\left[\begin{array}{cc}
\Delta & \Delta_{2} \\
\Delta_{3} & \Delta_{4}
\end{array}\right]\left[\begin{array}{cc}
N & 0 \\
0 & 0
\end{array}\right]\right)
\end{aligned}
$$

where $\mathcal{M}:=\left[\begin{array}{cc}M & 0 \\ 0 & 0\end{array}\right], \mathcal{L}:=\left[\begin{array}{ll}L & 0 \\ 0 & 0\end{array}\right]$, and $\mathcal{N}:=\left[\begin{array}{ll}N & 0 \\ 0 & 0\end{array}\right]$ are all square matrices with the same dimensions. It can easily be shown that

$$
\tau_{i}(M, L, N)=\tau_{i}(\mathcal{M}, \mathcal{L}, \mathcal{N})
$$

for $i=1, \ldots, \min (n, m)$. The proof is straightforward and involves showing that the minimum-norm perturbation to $(\mathcal{M}, \mathcal{L}, \mathcal{N})$ occurs at $\Delta_{2}=\Delta_{3}=\Delta_{4}=0$; the details are omitted.

1) Approximating a singular matrix with a nonsingular matrix: We used an approach similar to the singular value decomposition approach often used to approximate a matrix by one of lower rank (e.g. see [14]). In particular, let $M \in$ $\mathbb{C}^{n \times n}$ be a given singular matrix, and

$$
M=U \operatorname{diag}\left(\sigma_{1}, \ldots, \sigma_{n}\right) V^{*}
$$

be a singular value decomposition of $M$, where $U$ and $V$ are unitary, and $\sigma_{1}, \ldots, \sigma_{n}$ are the singular values of $M$, where $\sigma_{1} \geq \cdots \geq \sigma_{n}$. Since $M$ is singular, then there are one or more zero singular values; i.e $\sigma_{j}=\ldots=\sigma_{n}=0$. We approximated $M$ with $\tilde{M}$ by perturbing the zero singular values by a small amount, $\epsilon \ll 1$; i.e.

$$
\tilde{M}=U \operatorname{diag}\left(\sigma_{1}, \ldots, \sigma_{j-1}, \epsilon, \ldots, \epsilon\right) V^{*}
$$

It can be shown that $\|M-\tilde{M}\|_{2}=\epsilon$, and that the condition number $^{2}$ of $\tilde{M}$ is $\kappa(\tilde{M})=\sigma_{1} / \epsilon$. Hence, an advantage of this approach is its ease in choosing an approximation with a particular "distance" and/or condition number.

2) Results obtained: Interestingly enough, the approximation technique discussed above does not provide in general an estimate of a restricted real perturbation value that is close to the lower bound provided by Theorem 3.1. For example, consider the following matrix triplet:

$$
\begin{aligned}
M_{0}= & {\left[\begin{array}{cccc}
-5-1 i & 10-13 i & -16+4 i & 4+4 i \\
5-11 i & 6+7 i & -11+16 i & -6+19 i \\
13-1 i & 11+1 i & 7+33 i & -2+8 i
\end{array}\right] } \\
L_{0}= & {\left[\begin{array}{cccc}
-4-5 i & 16-11 i & -2-18 i & -5+6 i \\
-5+5 i & 10 & -3-11 i & 3+10 i \\
3+2 i & 7+1 i & -4 & -1+7 i
\end{array}\right] } \\
N_{0}= & {\left[\begin{array}{cccc}
10-2 i & -1-16 i & -14+4 i & -6+10 i \\
-6+13 i & 6+22 i & -6-9 i & 3-1 i \\
17+12 i & -7+1 i & -5-1 i & 2+7 i \\
-2 i & 12+5 i & -24+8 i & -1+9 i \\
8-11 i & -5+9 i & -4+8 i & -14-1 i
\end{array}\right] }
\end{aligned}
$$

Using the approximation technique outlined above with $\epsilon=$ $10^{-6}$, we obtained:

$$
\tilde{L}_{0}=\left[\begin{array}{cc}
-4-5 i & 16-11 i \\
-5+5 i & 10+6.83_{10^{-15}} i \\
3+2 i & 7+1 i \\
6.57_{10^{-7}} & -4.61_{10^{-7}}-3.43_{10^{-8}} i \\
-2-18 i & -5+6 i \\
-3-11 i & 3+10 i \\
-4+1.33_{10^{-15}} i & -1+7 i \\
1.84_{10^{-7}}-4.75_{10^{-7}} i & 7.26_{10^{-8}}+3.00_{10^{-7}} i
\end{array}\right]
$$

Table II shows the lower bounds of $\tau_{i}\left(M_{0}, L_{0}, N_{0}\right)$ provided by Theorem 3.1, and the estimates of $\tau_{i}\left(M_{0}, L_{0}, N_{0}\right)$ obtained by computing $\tau_{i}\left(\tilde{L}_{0}^{-1} \tilde{M}_{0}, N_{0}\right)$, where $\tilde{L}_{0}$ denotes an approximations of $L_{0}$, and $\tilde{M}_{0}$ equals $\left[\begin{array}{cc}M_{0} & 0 \\ 0 & 0\end{array}\right]$ such that $\tilde{M}_{0}$ conforms with the dimensions of $\tilde{L}_{0}$ and $N_{0}$. From Table II, we see that the estimates of $\tau_{i}\left(M_{0}, L_{0}, N_{0}\right)$ do not achieve the lower bounds. As mentioned earlier, this is generally the case. However, there are some cases where the lower bound is achieved, and these are presented below.

Consider the following two cases and examples:

Case 1: $L$ has full column rank; e.g.

$$
M_{1}=M_{0}, \quad L_{1}=\left[\begin{array}{cc}
-4-5 i & 16-11 i \\
-5+5 i & 10 \\
3+2 i & 7+1 i
\end{array}\right], \quad N_{1}=N_{0}
$$

${ }^{2}$ Assuming all the non-zero singular values of $M$ are greater than $\epsilon$. 
Case 2: $L$ is real; e.g.

$$
M_{2}=M_{0}, \quad L_{2}=\left[\begin{array}{cccc}
-4 & 16 & -2 & -5 \\
-5 & 10 & -3 & 3 \\
3 & 7 & -4 & -1
\end{array}\right], \quad N_{2}=N_{0}
$$

TABLE II

APPROXIMATION AND LOWER BOUNDS OF $\tau_{i}\left(M_{j}, L_{j}, N_{j}\right)$ FOR

$j=0,1,2$

\begin{tabular}{|c|c|c|c|}
\hline & $\begin{array}{c}\tau_{i}\left(\tilde{L}^{-1} \tilde{M}, N\right) \\
(\text { by }(23))^{*}\end{array}$ & $\begin{array}{c}\tau_{i}(M, L, N) \\
(\text { by Theorem 3.1)* }\end{array}$ & $\sigma_{i}(M, L, N)$ \\
\hline \multicolumn{4}{|c|}{ Case 0: $\left(M_{0}, L_{0}, N_{0}\right)$} \\
\hline$i=1$ & 15345 & 15339 & 0.19336 \\
\hline 2 & 1912.3 & 0.076982 & 0.073709 \\
\hline 3 & 0.027633 & 0.023693 & 0.018815 \\
\hline \multicolumn{4}{|c|}{${ }^{* *}$ Case 1: $\left(M_{1}, L_{1}, N_{1}\right)$} \\
\hline$i=1$ & $8.5355_{10+10}$ & $\infty$ & $\infty$ \\
\hline 2 & 1073.3 & 1073.3 & 0.095529 \\
\hline 3 & 0.038480 & 0.038480 & 0.026681 \\
\hline \multicolumn{4}{|c|}{${ }^{* *}$ Case 2: $\left(M_{2}, L_{2}, N_{2}\right)$} \\
\hline$i=1$ & 20822 & 20822 & 0.38061 \\
\hline 2 & 204.70 & 204.70 & 0.11524 \\
\hline 3 & 0.033333 & 0.033333 & 0.029250 \\
\hline
\end{tabular}

* For numerical purposes, the search in (23) and (11) is in $\gamma \in\left[10^{-5}, 1\right]$. ${ }^{* *}$ In these cases, the lower bounds obtained by Theorem 3.1 are very close to the approximated values (see bold values).

From Table II, we see that when $L$ has full column rank and/or is real, the estimates of the restricted real perturbation values of $(M, L, N)$ obtained by $\tau_{i}\left(\tilde{L}^{-1} \tilde{M}, N\right)$ are very close to the lower bounds provided by Theorem 3.1 (shown in the second column). From experiment, this seems to be true for any $L$ with full column rank and/or is real, and not just for the particular examples shown here. Therefore this suggests that for these two cases, the lower bound is actually achievable; i.e. equality in (11) holds. The same can be said about $N$ with full row rank and/or is real.

Table II also lists $\sigma_{i}(M, L, N)$, which is another lower bound (see Remark 3.2) for the restricted real perturbation values of $(M, L, N)$. We see that the lower bounds provided by Theorem 3.1 are much tighter than $\sigma_{i}(M, L, N)$, and in some cases, the differences are quite large.

\section{Conclusions}

In this paper, we further generalized the real perturbation value problem of [1] to account for more general perturbation structures and presented a formula for computing lower bounds of the so-called restricted real perturbation values of a complex matrix triplet $(M, L, N)$. Using these results, we revisited the pendulum problem in [11] and obtained the true value of the structured real controllability radius of the multi-link inverted pendulum system. In the future, the restricted real perturbation values can also be applied to make extensions, similar to that made to the structured real controllability radius, to the other LTI robustness measures (e.g. the structured real decentralized fixed-mode radius, the structured real minimum-phase radius, etc.).
This paper also studied the exactness of the lower bounds provided by Theorem 3.1, which are proved to be tighter than simply computing the restricted singular values of $(M, L, N)$. In particular, using approximation techniques, we experimentally showed that the lower bounds provided by Theorem 3.1 are actually achievable when $L$ (or $N$ ) have full column (or row) rank, and/or is real. In fact, it can readily be shown that when $(L, N)=(I, I)$ or $L=I$, the formula (11) for computing the lower bound of $\tau_{i}(M, L, N)$ reduces to the formulas for computing the ordinary and generalized real perturbation values respectively (i.e. from Theorem 2.1 and [6]) where equality actually holds:

$$
\begin{aligned}
\tau_{i}(M, I, I) & =\tau_{i}(M)=\sup _{\gamma \in(0,1]} \sigma_{2 i-1}\left(M_{\gamma}^{\mathbb{R}}\right) \\
\tau_{i}(M, I, N) & =\tau_{i}(M, N)=\sup _{\gamma \in(0,1]} \sigma_{2 i-1}\left(M_{\gamma}^{\mathbb{R}}, N_{\gamma}^{\mathbb{R}}\right)
\end{aligned}
$$

As equality holds in both of these two cases, this leads one to conjecture that equality should also hold in Theorem 3.1. A more analytical study investigating the achievability of the lower bound in Theorem 3.1 for the case when $L$ (or $N$ ) have full column (or row) rank, and/or is real, and also for the general cases of $L$ and $N$, is currently being conducted by the authors.

\section{REFERENCES}

[1] B. Bernhardsson, A. Rantzer, and L. Qiu, "Real perturbation values and real quadratic forms in a complex vector space," Linear Algebra and its Applications, vol. 270, pp. 131-154, 1998.

[2] G. Hu and E. J. Davison, "Real controllability/stabilizability radius of LTI systems," IEEE Trans. Automat. Contr., vol. 49, no. 2, pp. 254257, 2004.

[3] S. Lam and E. J. Davison, "The real decentralized fixed mode radius of LTI systems," in $46^{\text {th }}$ IEEE Conference on Decision and Control, New Orleans, LA, USA, December 12-14 2007, pp. 3036-3041.

[4] — - "The transmission zero at $s$ radius and the minimum phase radius of LTI systems," in $17^{\text {th }}$ IFAC world congress, Seoul, Korea, July 6-11 2008, pp. 6371-6376.

[5] L. Qiu, B. Bernhardsson, A. Rantzer, E. J. Davison, P. M. Young, and J. C. Doyle, "A formula for computation of the real stability radius," Automatica, vol. 31, no. 6, pp. 879-890, 1995.

[6] S. Lam and E. J. Davison, "Generalized real perturbation values with applications to the structured real controllability radius of LTI systems," in 2009 American Control Conference, St. Louis, Missouri, USA, June 10-12 2009, pp. 2439-2444.

[7] C. V. Loan, "Generalizing the singular value decomposition," SIAM J. on Numerical Analysis, vol. 13, no. 1, pp. 76-83, 1976.

[8] C. C. Paige and M. A. Saunders, "Towards a generalized singular value decomposition," SIAM J. on Numerical Analysis, vol. 18, no. 3, pp. $398-405,1981$.

[9] H. Zha, "The restricted singular value decomposition of matrix triplets," SIAM J. on Matrix Analysis and Applications, vol. 12, no. 1, pp. 172-194, 1991.

[10] B. L. R. De Moor and G. H. Golub, "The restricted singular value decomposition: Properties and applications," SIAM J. on Matrix Analysis and Applications, vol. 12, no. 3, pp. 401-425, 1991.

[11] S. Lam and E. J. Davison, "The real stabilizability radius of the multi-link inverted pendulum," in 2006 American Control Conference, Minneapolis, MN, USA, June 14-16 2006, pp. 1814-1819.

[12] D. Hinrichsen and A. J. Pritchard, Mathematical Systems Theory I: Modelling, State Space Analysis, Stability and Robustness. SpringerVerlag, Berlin, 2005.

[13] G. H. Golub and C. F. V. Loan, Matrix Computations. The Johns Hopkins University Press, 1983.

[14] G. W. Stewart, "On the early history of the singular value decomposition," SIAM Review, vol. 35, no. 4, pp. 551-556, 1993. 